\title{
Folding a neuroscience center into streamlined COVID-19 response teams
}

\section{Lessons in origami}

Daniel J. Correa, MD, MSc, Daniel L. Labovitz, MD, Mark J. Milstein, MD, Renee Monderer, MD, and Sheryl R. Haut, MD, MSc

Neurology ${ }^{\circledR}$ 2020;95:583-592. doi:10.1212/WNL.0000000000010542

\author{
Correspondence \\ Dr. Correa \\ daniel.correa@ \\ einsteinmed.org
}

\section{MORE ONLINE}

\section{COVID-19 Resources}

For the latest articles, invited commentaries, and blogs from physicians around the world

NPub.org/COVID 19

From the Saul R. Korey Department of Neurology (D.J.C., D.L.L., M.J.M., R.M., S.R.H.), Montefiore Medical Center; Comprehensive Einstein/Montefiore Epilepsy Center (D.J.C., D.L.L., S.R.H.), Montefiore Medical Center; Stern Comprehensive Stroke Center (D.L.L.), Montefiore Medical Center; Department of Medicine (Critical Care) (D.L.L., M.J.M., S.R.H.), Montefiore Medical Center; Albert Einstein College of Medicine (D.J.C., D.L.L., M.J.M., R.M., S.R.H.), Bronx, NY.

Go to Neurology.org/N for full disclosures. Funding information and disclosures deemed relevant by the authors, if any, are provided at the end of the article. 
The Montefiore Neuroscience Center, within a large Bronx hospital network, recently faced the challenge of providing clinical neurologic care in the epicenter of the COVID-19 pandemic $^{1}$ to a population at high risk for Covid-19 illness morbidity and death. Our department was faced with multiple pressing issues at the onset of the surge, which was an entirely unique medical experience and included the following: an immediate need for obtaining personal protective equipment (PPE) and developing safety protocols, dramatic reductions to our staffing because of redeployment to frontline COVID-19 care, and the rapid need to recognize and treat novel manifestations of neuro-COVID. These priorities occurred nearly simultaneously and necessitated rapid, often daily, changes to our protocols and practices. Herein, we describe how, over 3 weeks, we transformed our clinical services by reducing neuroscience beds from 4 wards to just 1, while delivering care to over 600 hospitalized patients with neuro-COVID and over 1,742 total neuroscience hospital bed days. We share these experiences as other centers may now face similar challenges with their initial surge or potential second waves and as we transition to a postsurge neurology service that will remain changed, perhaps permanently. 2,3

In adapting our services during the COVID-19 crisis, we focused on 4 planning targets: (1) strategies to mitigate exposure and transmission, (2) protection of the health and safety of staff, (3) alleviation of logistical delays and strains on the system, and (4) facilitating coordinated communication. Health systems should not wait until they face a surge in COVID-19 cases to implement a comprehensive response. By acting early, health systems may avoid being crippled by crisis and continue to provide critically important care.

\section{Pre-COVID-19 neurology services}

Within the Montefiore Health System, the Neurology Department assumes direct care of neurology inpatients and consultations at the 3 Montefiore Legacy Hospitals: Montefiore Medical Center (Moses campus), Jack D. Weiler Hospital at the Albert Einstein College of Medicine, and Montefiore Medical Center Wakefield. Routinely, we also accept stroke and neurology transfers from a network of 6 hospitals within the Montefiore Health System and 2 regional hospitals outside the system.

The inpatient neuroscience program at the Moses campus, which is a Joint Commission-certified comprehensive stroke center and level 4 comprehensive epilepsy center, comprises a 10-bed neuroscience ICU (NSICU), a 15-bed neuroscience progressive care (stepdown) unit (NSPCU), a 4-to-6-bed adult epilepsy monitoring unit, and 24 neuroscience beds located in 2 hospital wards. The Jack D. Weiler Hospital campus includes inpatient neurology beds, consult neurology, medical ICUs, and inpatient EEG sciences. At the Wakefield campus, our neuroscience services include consult neurology and EEG services.

\section{COVID-19 transition}

The first reported case of COVID-19 in New York State was confirmed on March 1, 2020, within Manhattan. Soon afterward, the first patient with known COVID-19 was admitted to Montefiore Medical Center on March 9, 2020, and in figure 1, we describe the critical transition points in the timeline after the first admission.

On March 12, we formed a COVID-19 neurology leadership task force. This group consisted of clinical leaders representing Stroke, Neurohospitalists, Epilepsy, and Pediatric Neurology, and our Service Chiefs, Residency Director, Department Chair, and Unified Administrator. Figure 2 shows the daily census trend at the Montefiore Medical Center compared with NY State COVID-19-positive cases demonstrating the surge of cases in the region. The early formation of the leadership task force with meetings by telephone 7 days per week allowed us to make decisions quickly.

\section{Strategies to mitigate exposure and transmission}

\section{Limit those entering our health care facilities}

The neurology department joined the Montefiore Hospital System in redeploying staff to reduce the number of individuals entering the hospital and clinics to mitigate potential nosocomial spread of COVID-19. ${ }^{4}$ We consolidated all inpatient neuroscience services from all 3 hospitals to the Montefiore Medical Center (Moses campus), whereas the other 2 legacy hospitals maintained consultation (telemedicine and as-needed in-person) services. A list of departmental staff and house staff with high-risk conditions (hypertension, diabetes, immunosuppression, and/or age $>65$ years or an immunosuppressed cohabitant) was identified privately by self-report to designated senior leadership. Highrisk staff were removed from the patient contact care and placed into a pool for remote clinical, administrative, and teaching services. One neurology resident was excluded, and another individual's schedule and roles were adjusted to accommodate for high-risk screening conditions.

To further reduce exposure, our hospital system excluded all visitors, with rare, prespecified, exceptions (such as nonCOVID end-of-life care). All logistics and department meetings were converted to virtual meetings. Educational sessions became teleconference lectures and conferences. Clinical clerkships for students were canceled, and we initiated the design of a new neurology clinical clerkship adapted to the new environment starting July 1, 2020.

Our epilepsy monitoring unit was closed, and elective neurosurgeries were canceled on March 16. These changes reduced exposure of patients to the hospital as COVID-19 admissions began increasing. The reduction in elective admissions provided additional inpatient beds in the neuroscience unit to house the increasing volume of patients with stroke transferred from network and outside hospital sites.

The stroke service was the first division to reorganize, designating teams to cover the inpatient stroke service, hospital 
Figure 1 Timeline of critical transition points

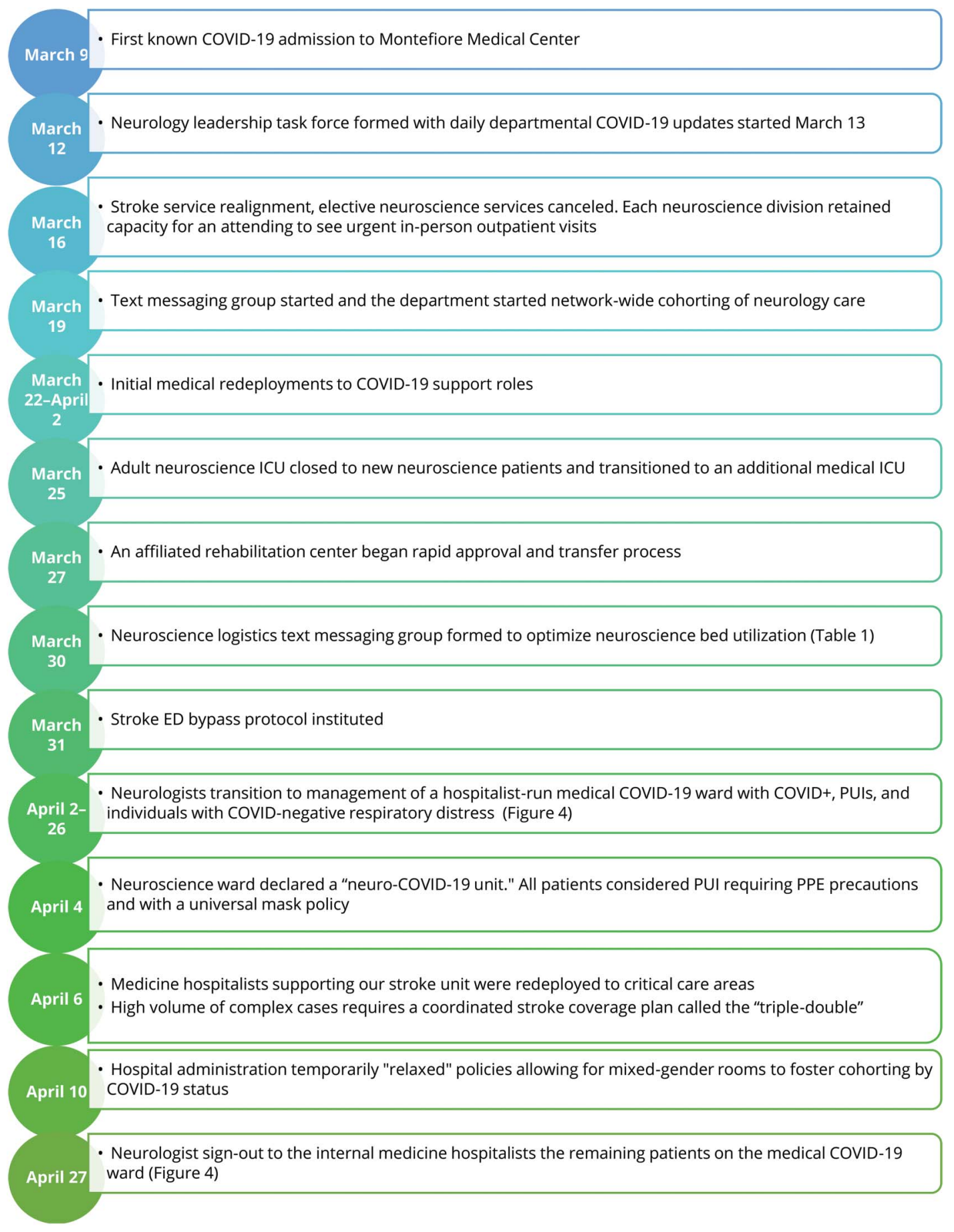

consult, and outpatient teams. The hospital consult team covered requests for transfer from outside hospitals, emergency department consults including orchestration of tPA and mechanical thrombectomy, and inpatient stroke follow-ups via electronic consult (E-consult). For emergency department (ED) and inpatient consults, the patient history was obtained by the neurology consult resident; chart review was performed by the resident and consult attending; and physical examinations were performed by either the primary team or a neurology resident, as needed. All outpatient stroke center visits were transitioned to telehealth.

Mirroring the stroke division, the size of all in-person clinical teams was reduced. Additionally, staff with high-risk medical conditions were excluded from direct patient care. Where possible, teams conducted rounds virtually, and met in person only when necessary for patient care. When feasible, attendings performed any bedside procedures to limit exposure of house staff and physician assistants 


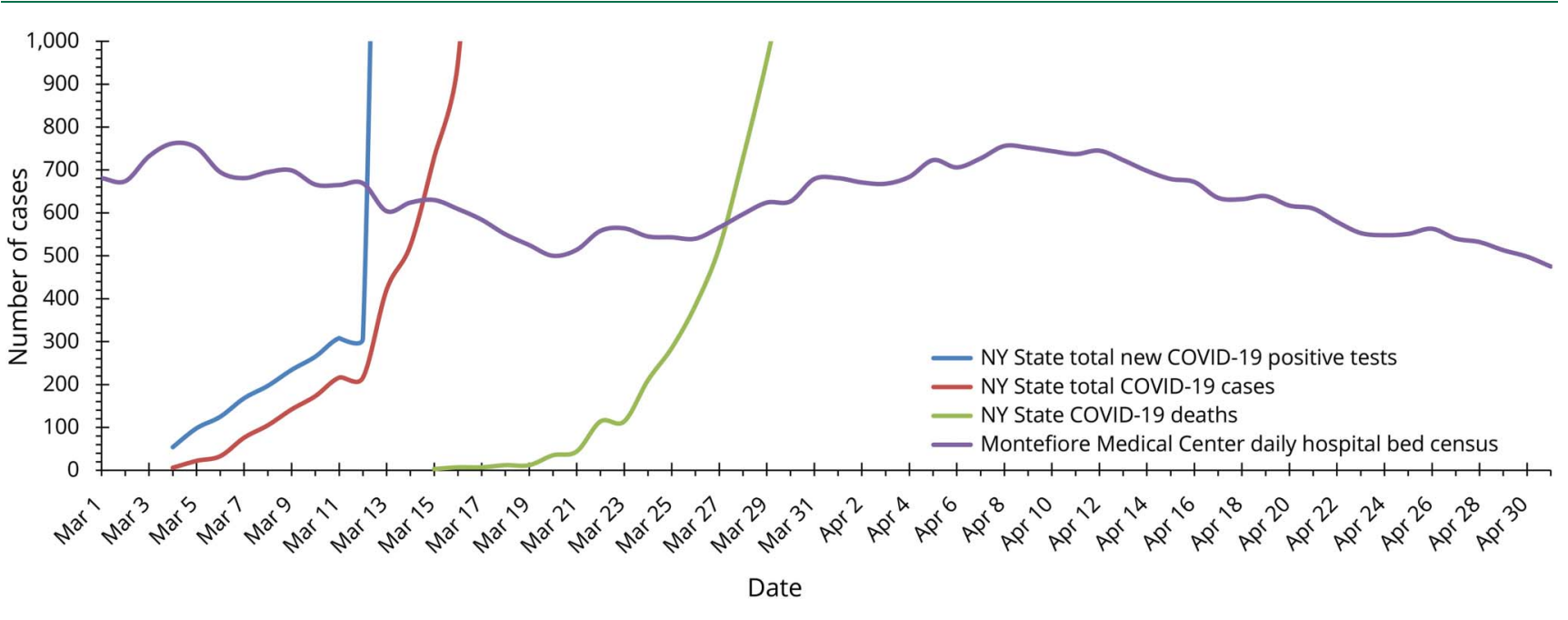

and to conserve PPE. This system required the development of a robust E-consult neurology service. To facilitate E-consult, templates were developed for our electronic medical record. The Econsult team was staffed with experienced residents (postgraduate year 3-5) going to the bedside when necessary. Supervision and teaching was staffed remotely by at-risk faculty to shield them from patient-facing care. In rare situations, inpatient attendings were used as backup to answer more complex bedside questions.

Early hurdles included frequently rotating team members with increased handoffs, working longer shifts, and limited access to diagnostic testing (such as MRI, EEG, and nerve conduction studies) to reduce nosocomial transmission. Neurodiagnostic testing protocols where adapted to protect technicians and staff when medically necessary $\mathrm{EEG}^{5}$ and nerve conduction studies were requested. Increased attention to direct sign-out and formal communication with other divisions helped reduce the risk of multiple handoffs. To decrease the burden of increased hours, our hospital system and department supported staff with free parking, free meals, reimbursed travel, and partnerships with regional hotels.

We deployed telemedicine resources using phone or video conferencing at the preference of the individual/family to avoid unnecessary exposure while still providing robust outpatient neurology care. ${ }^{2,4,6}$ This was facilitated on March 17 when the Centers for Medicare \& Medicaid Services announced expanded Medicare coverage for telemedicine. ${ }^{7,8}$ Our model was similar to the virtual neurology plan described by Grossman et $\mathrm{al}^{9}$ and included history, a modified virtual examination, coding, and billing. In addition, our stroke team colleagues developed a rapid adapted stroke assessment early in the response period. ${ }^{10}$ At this stage, we maintained outpatient neurology care by identifying patients for whom an in-person visit was essential. In addition, the outpatient case managers and social workers continued their roles through telephone or telemedicine services.
We defined essential patients as those with refractory conditions that could not be managed remotely and that potentially required admission to the hospital. We arranged video visits when possible, but some patients lacked internet access or were uncomfortable or unable to use the technology needed for video visits. Therefore, most encounters, especially early on, were conducted by telephone without video. These visits were received well, often with relief, by patients worried about the risk of exposure in a doctor's office. Many, particularly those with childcare responsibilities or limited mobility, preferred the convenience of in-person visits. On April 1, our hospital implemented an HIPAA-compliant video telehealth system, which made it easier for a rising proportion of patients to access video visits as this system has improved. As the number of patients with COVID in the New York area dropped, the small number of patients uncomfortable with telehealth services could schedule in-person visits.

\section{Reducing essential personnel}

The department identified nonfrontline health care workers who could transition to remote support, or those whose standard responsibilities were substantially reduced, and assigned them support roles for our teams. In our department, we defined nonfrontline staff as departmental administrative staff and outpatient clinic secretaries. Team members who were not supporting a telehealth service remotely were each given remote tasks to support house staff education programs and departmental administrative tasks.

\section{Protection of the health and safety of staff}

The use of PPE (including procedure face masks, N95 masks, eye protection/face shields, nonsterile nitrile gloves, and isolation gowns) for health care personnel providing care during this crisis was vital for the safety of patients and caregivers. In addition to hospital resources, neurology department leaders acquired additional equipment from external sources, typically donation based, to ensure availability of PPE for our personnel to provide care safely. Our staff were updated regularly, sometimes daily, on how 
to access to hospital PPE supplies and additional back-up supplies available from the department during hospital shortages.

\section{Tactics to alleviate logistical delays and strains on the system}

\section{Stroke cohorting}

On March 19, we initiated network-wide stroke cohorting, with automatic transfer of all ED patients with stroke from our legacy hospitals to our inpatient stroke service. This increased efficiency and reduced wait time for beds at the other legacy hospitals.

\section{Hospital redeployments}

Simultaneous to stroke cohorting, our department underwent significant redeployment of neurology attendings, fellows, and residents to COVID-19 respiratory illness work, including ED screening tents (March 22), a COVID-19 respiratory illness Med-Surg ward (housed in the previous attending-only neurology ward) (March 29), and multiple COVID-19 ICUs (April 2).

The Montefiore Health System administration, on March 20, asked each department to provide a list of available staff for redeployment. Departments were assigned various deployment assignments throughout the hospital. Given the extensive experience of neurologists with critically ill patients, neurology was one of the few nonmedicine departments to be selected for deployment to COVID-19 ICU work in addition to staffing the COVID-19 tents and a medical COVID-19 ward.

\section{Deployment staffing model}

$50 \%$ of neurology residents, fellows, and nonexempt faculty were needed for staffing a full Med-Surg COVID-19 unit and helping staff multiple COVID-ICUs. Junior residents (PGY13 ) were used for redeployment, and senior residents and fellows (PGY4-6) were used for barebones staffing of our consultation and inpatient units. Using this model, our consult services could have supervisory attendings working almost exclusively remotely, and in-person attending evaluations were the exception rather than the rule. However, limited house staff for our inpatient services increased the attending burden for high-volume services such as stroke.

Within the NSICU, outside rotators from a local emergency medicine residency stopped completely a few weeks into March. The critical care fellows normally on service throughout the week were often redeployed to other ICU support roles. Rotators from neuroscience specialties were also not available to the NSICU critical team during the emergency redeployments.

The hospital mobilized a majority of its beds for respiratory illnesses, whereas the neurology department joined a select group of services (psychiatry and oncology) in the system, proposed a staffing organization to maintain some inpatient specialty services, and provided a list of available clinical staff for redeployment. Although the stroke faculty possessed skills useful in an ICU, they were not redeployed but took over roles previously staffed by the general neurology faculty and medicine hospitalists, including management of a census of stroke patients with higher acuity than usual, with more transfers from outside hospitals and immediate transfer to the NSPCU after thrombectomy.

To support redeployed nonmedicine clinical staff, our critical care and internal medicine colleagues provided webinars and orientation sessions and an internal document with suggestions for COVID-19 management of respiratory distress, pneumonias, electrolytes disturbances, acid-base disorders, acute kidney injuries, and monitoring inflammatory markers for possible cytokine storm. This was complemented by on-site support via a rapid E-consult process with an on-call medicine hospitalist COVID-19 advisory team, including our nephrology, hematology, infectious disease, and critical care colleagues.

On March 25, the NSICU was closed to neuroscience ICU patients and reopened as an extension of medical ICU space. NSICU patients without COVID-19 were housed in the postanesthesia care unit, with increased pressure to bring these patients to the NSPCU, typically after 2 hours of recovery, per protocol.

Our ICUs were not formally converted to Medical COVID19 critical care units; rather, the ICU case load required a major expansion of ICU bed capacity. The most critically ill patients received the ICU beds. During the peak of the crisis, this happened to be almost entirely patients with COVID-19; however, a small number of patients without COVID continued to be admitted to the ICUs as well. At the Montefiore Medical Center, our hospital and critical care leadership added ICU capacity by converting a former medicine ward, a pediatric ward, and a postacute care unit in ICU beds. In addition, an off-site ambulatory surgery center was converted to accommodate surge capacity of ICU and medicine beds. The administration also explored conversion of the operating rooms to ICU beds; however, that area was closed only after admitting 1 patient and a decline in cases.

\section{Nursing and clinical support staff}

For the most part, the nursing staff stayed with their units; some neuroscience nurses were called upon to help ICU nursing when additional staffing was needed. Some staff in the hospital nursing float pool also supported the ICU nursing as helpers. Many outpatient and ambulatory surgery center nurses were redeployed to inpatient areas based on their skill set. Some nursing staff who had been designed as higher risk were tasked as educators to train and orient newly arrived nursing graduates as helpers during the crisis.

\section{Rapid discharge to rehabilitation}

Case managers and social workers maintained their focus on transitioning patients out of the hospital with all rehabilitation 
Table 1 Seven types of neuroscience ward beds at Montefiore Medical Center

\begin{tabular}{ll}
\hline $\begin{array}{l}\text { Bed } \\
\text { type }\end{array}$ & Montefiore Medical Center neuroscience ward bed types \\
\hline $\mathbf{1}$ & Floor non-COVID \\
\hline $\mathbf{2}$ & Floor COVID \\
\hline $\mathbf{3}$ & Floor crash bed (single rooms) \\
\hline $\mathbf{4}$ & $\begin{array}{l}\text { Neuroscience progressive care (stepdown) unit (NSPCU) non- } \\
\text { COVID }\end{array}$ \\
\hline $\mathbf{5}$ & NSPCU COVID \\
\hline $\mathbf{6}$ & NSPCU crash bed (single rooms) \\
\hline $\mathbf{7}$ & Negative pressure rooms \\
\hline
\end{tabular}

and long-term care facilities receiving patients. On March 27, Burke Rehabilitation Center, an affiliated primary acute rehabilitation center for our health system, began accepting patients with COVID-19 and instituted a relaxed insurance approval process without rehabilitation medicine screening. This critically reduced the length of stay, which effectively increased unit capacity and improved flow through the neuroscience unit.

\section{ED bypass protocol}

On March 31, we launched an ED bypass protocol modeled on the pathway to mechanical thrombectomy, where patients with stroke with acute large vessel occlusions travel directly from CT to angiography suite to the NSICU without entering the ED. In the bypass protocol, all patients with stroke arriving directly to Moses traveled straight from CT to our stroke unit, where they received all laboratory testing and acute evaluation previously performed in the ED. Patients arriving from EDs of other hospitals also went directly to the stroke unit without passing through the Moses ED. For this model to be effective, singleroom crash beds capable of accepting patients with unknown COVID status had to be available at all times. Two single-room negative pressure rooms were reserved for patients requiring aerosolizing procedures or oxygen via a high-flow nasal cannula.

\section{Availability of disease-modifying care interventions}

Thankfully, our health system's acquisition of additional emergency ventilator equipment allowed for sufficient airway support options in the areas covered by our neuroscience colleagues. System wide, we did encounter a shortage of highflow nasal cannula equipment; however, any acute respiratory decompensation was managed in coordination with our critical care teams, and all patients who required ventilator support received care as indicated by their code status. There were no new ethical issues or change in the protocol for determination of code status.

As COVID-19 management evolved, the recommend treatment algorithms included placing a substantial portion of patients with COVID-19 on anticoagulation (mostly factor $\mathrm{Xa}$ inhibitors). The day after this recommendation was disseminated to hospital staff, there was a shortage for several hours as our pharmacies reassessed the volume of need. Within 12 hours, the supplies for factor Xa inhibitors were restored and future orders were adjusted accordingly. This experience resulted in hospital and department leadership, considering other support services that should be included in management planning.

In our stroke interventions, standards and protocols for mechanical thrombectomy did not change, and COVID status,

Table 2 Pre-COVID-19 and COVID surge neuroscience clinical care areas at Montefiore Medical Center

\begin{tabular}{|c|c|c|c|c|}
\hline $\begin{array}{l}\text { Montefiore Medical Center } \\
\text { neuroscience teams }\end{array}$ & No. of beds & $\begin{array}{l}\text { Clinician count } \\
\text { (roles) }\end{array}$ & House staff count (roles) & COVID surge teams \\
\hline $\begin{array}{l}\text { General } \\
\text { neurology-attending-only } \\
\text { neurohospitalist }\end{array}$ & $12-14$ & 2 (attending and PA) & 0 & $\begin{array}{l}1 \text { Attending and } 1 \text { PA on } \\
12 \text {-h shifts }\end{array}$ \\
\hline $\begin{array}{l}\text { General neurology-teaching } \\
\text { attending service }\end{array}$ & $\begin{array}{l}\text { Flexible floor beds } \\
\text { along with NSPCU } \\
\text { beds }\end{array}$ & 1 & $\begin{array}{l}3 \text { (Chief, Neuro PGY2, and Psych } \\
\text { PGY1) }\end{array}$ & \multirow{3}{*}{$\begin{array}{l}\text { Combined teaching, } \\
\text { neurology and stroke } \\
\text { teams } \\
\text { Stroke attending, } 1 \\
\text { neurology resident, } 1 \text { PA }\end{array}$} \\
\hline Vascular/stroke neurology & $\begin{array}{l}\text { Flexible floor beds } \\
\text { along with NSPCU } \\
\text { beds }\end{array}$ & 1 & $\begin{array}{l}5 \text { (Fellow, Chief, Neuro PGY2, and } 2 \\
\text { medicine PGY2) }\end{array}$ & \\
\hline $\begin{array}{l}\text { Neuroscience progressive } \\
\text { care (stepdown) unit (NSPCU) }\end{array}$ & 15 & $\begin{array}{l}2 \text { (vascular attending } \\
\text { and Med-hospitalist) }\end{array}$ & See stroke team & \\
\hline Epilepsy monitoring unit & $4-6$ & 1 & 1-2 (Fellow and Neuro PGY2/3) & 1 Attending and 1 fellow \\
\hline Neurosurgery & $4-8$ & 2 (attending and NP) & $\begin{array}{l}\text { 7-9 (PGY1, } 2 \text { PGY2, PGY3, } 2 \text { PGY4, } 2 \\
\text { PGY6, and Chief PGY7 }\end{array}$ & $\begin{array}{l}1 \text { Attending, } 1 \mathrm{NP} \text {, and 1-2 } \\
\text { neurosurgery residents }\end{array}$ \\
\hline Neuroscience ICU (NSICU) & 10 & 1 & $\begin{array}{l}\text { 1-2 (Fellow, intermittent } \\
\text { neurosurgery intern, neurology } \\
\text { resident) }\end{array}$ & $\begin{array}{l}1 \text { attending with staff } \\
\text { redeployed from other } \\
\text { areas }\end{array}$ \\
\hline
\end{tabular}




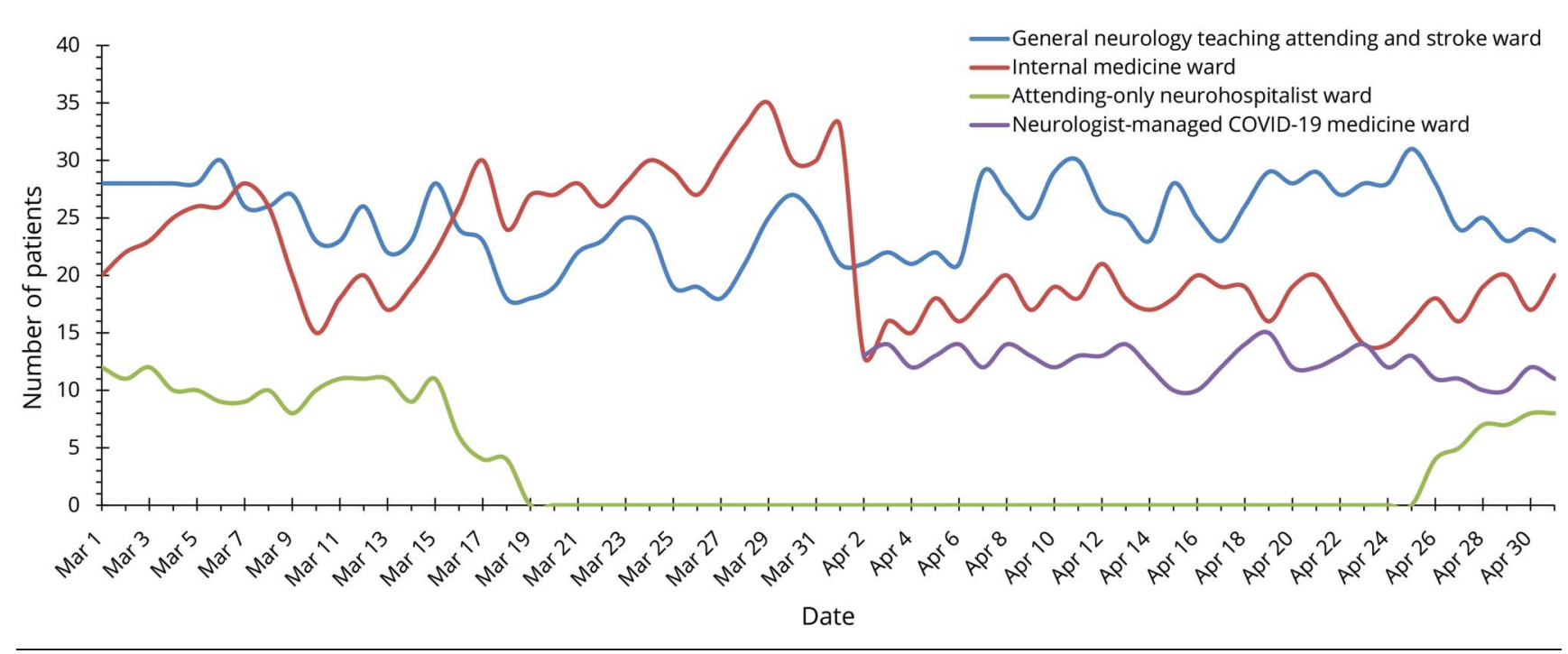

which was often unknown when treatment began, did not affect eligibility. There was no rationing of disease-modifying stroke care including tPA and mechanical thrombectomy.

\section{Facilitating coordinated communication}

A daily departmental email COVID-19 update was sent out starting March 13. Daily updates included the following sections: general hospital updates, neurology-specific clinical updates, COVID-19 protocol/treatment updates, PPE availability, training/education updates, and wellness corner.

A text group using popular CMS-approved encrypted texting software ${ }^{8,11}$ was formed on March 19 to facilitate movement of neuroscience patients. Members included stroke, general neurology, neurosurgery and medicine hospitalist attendings, stroke fellows, rotating residents, a charge physician assistant,

Figure 4 Stroke team triple-double surge coverage plan with 4 attendings and 2 fellows

7-day shift "Green team"

- Day: Stroke attending

"G1" and "G2"

- Night: Stroke fellow

"F2"

\section{7-day shift "Blue team" \\ - Day: Stroke attending \\ "B1" and "B2" \\ - Night: Stroke fellow \\ "F1"}

7-day shift "Blue team"

- Day: Stroke attending

"B1" and "B2"

- Night: Stroke fellow

"F1" 
and the nurse manager. Immediate communication for urgent clinical updates, bed management, and triage were established. On March 30, a neuroscience logistics texting group was formed to optimize use of 7 types of neuroscience ward beds (table 1) among our pre-COVID-19 clinical neuroscience areas and our COVID surge teams (table 2). The easy acceptance by hospital nurses, physicians, and physician assistants of familiar texting software ${ }^{8,11}$ was a key factor in our neuroscience transition. Although hospital-generated digital communication formats existed, the flexibility and familiarity of the software, including the ability to reach large groups and rapidly add and remove staff, was critical.

\section{Formation of a neuro-COVID-19 neuroscience unit and transition to medicine COVID unit support}

The general neurology teaching service, epilepsy monitoring beds, neurosurgery, and stroke unit were converted to a neuro-COVID-19 unit on April 4. Before this date, patients were transferred to non-neurology COVID-19 medical wards once a diagnosis of COVID-19 was established. The large number of COVID-19-positive cases of with stroke, seizure, and encephalopathy, combined with the reduced number of non-COVID-19 neuroscience admissions, drove this change.

On April 2, a team of neurologists transitioned to management of up to 14 beds on an internal-medicine-hospitalistmanaged COVID-19 ward. Figure 3 shows the neuroscience inpatient daily census trends from March 1, 2020, to May 1, 2020 .

Over a 30-day period, the neurologist-managed COVID-19 medicine ward included patients with COVID-related respiratory illnesses, along with patients pending SARS-CoV2 tests, and COVID-negative patients. During this period, our redeployed colleagues cared for a daily census ranging from 11 to 15 patients, of which one- to two-thirds were confirmed COVID positive.

On April 6, the medicine hospitalists who support our stroke unit were redeployed to other critical care areas. The high medical acuity of the neuroscience cases required a coordinated stroke team coverage plan, which we called the triple-double. Teams consisted of 3 work pairs working 7 on/ 7 off: 2 stroke attendings covering the day, with 1 stroke fellow covering in-house at night (figure 4).

\section{Lessons learned}

Our recent experience is informative for neuroscience centers preparing for any potential future emergency response during this or future public health emergencies and may be generalized across several specialty disciplines. The keystones of our experience include rapid adaptation, synchrony across divisions, maximizing bed capacity while preserving resources, administrative flexibility, and excellent digital communication.

\section{Rapid adaptation and flexibility}

As evidenced by the timeline in figure 1, our neuroscience center underwent nearly continuous change over a 3-week period of the COVID-19 surge. The elements that contributed to this adaptation included daily leadership calls, daily departmental emails, and continuous communication via text. A portion of the leadership calls was devoted to identifying the points for the daily departmental email updates, as these served a critical piece of departmental communication and flexibility. The announcements, open airing of questions, and discussion of neurology redeployment to COVID-19 medical support were all essential in reducing anxiety and promoting cohesiveness during this highly stressful chapter.

\section{Rapid synchrony across divisions}

Although our department previously operated in a more typical silo model, that model was entirely impractical during the public health emergency. As has been already reported, the neurologic manifestations of COVID-19 $9^{12-15}$ were often nonspecific and across disciplines. For example, a patient presenting with aphasia/altered sensorium might initially present as a stroke code, quickly be reclassified as possible COVID-19 encephalopathy, and subsequently be found to be in nonconvulsive status epilepticus. The seamless and immediate communication between the stroke, general neurology, neurophysiology, and pediatric neurology divisions ensured that the potentially neuro-COVID-19 population was treated aggressively and efficiently at all times. Furthermore, frequent deployment adjustments necessitated restaffing wards and services across typical division boundaries.

\section{Maximizing bed capacity while preserving resources}

As the surge hit our network situated in the epicenter of COVID-19 cases in NYC, it became quickly evident that the neurologic complications of COVID-19 would result in a large volume of inpatients with primary neurologic deficits. The immediate steps taken to maximize bed capacity and cohort neurology patients from across our network enhanced our ability to preserve our precious resources: staff and PPE. The formation of a neuro-COVID-19 unit allowed us to optimize our use of nondeployed staff and housing patients with neuro-COVID-19 on one floor allowed staff to maximize the use of PPE.

\section{Reduced dependence on diagnostic testing}

The large number of admitted patients with COVID-19 made overreliance on diagnostic testing impractical. CT was readily available for all of our patients as rooms could be rotated to facilitate cleaning in between patients. However, the use of MRI and electrophysiologic testing became extremely limited. MRI was frequently deferred when pathology was clearly delineated on CT, although a limited number of patients were able to be evaluated when it was important to their 
management. Each EEG request was triaged by the epilepsy group, and single-use 8-channel EEG headbands (Ceribell Rapid Response EEG) were used instead of standard EEG machines wherever possible. EMGs were performed only where crucial, predominantly to assess patients for acute inflammatory demyelinating polyneuropathy thought to be triggered by infection and brachial plexus injuries presumed related to patient proning. Real-time triaging of test importance rather than rote diagnostic workups was necessary to maintain patient flow and bed availability. This strategy also served to limit extended hospital exposure for COVID-negative patients.

\section{Administrative flexibility}

The medical center's response as a major COVID-19 epicenter facility was instrumental toward successful navigation. Each request indicated above was immediately cleared by hospital administration, often forgoing the usual channels, in an effort to achieve rapid and smooth change. This flexibility was well demonstrated by the ED bypass protocol developed and implemented in the course of 1 day after a conversation between the Director of Emergency Medicine and the Comprehensive Stroke Center Director.

Although we have passed the initial COVID-19 surge in NYC, the lessons learned remain fresh in our minds. Throughout the international medical community, health care leaders have described their experiences and recommendations as we adapt to meet the demands of this crisis. ${ }^{9,16-22}$ As we are slowly transitioning to the next phase of medical care in this COVID-19 era, we remain highly cognizant that a similar crisis may recur at any point. Our department has, therefore, elected to continue many of the changes already instituted for the foreseeable future. Telemedicine and E-consults are now elements of our armamentarium of care. Frequent updates by email, teleconferences, and text messaging groups have remained essential in this new era. Based on our experience, clinical leaders should not delay planning a COVID-19 response framework and consider acting now to develop adaptive and systematic approaches consistent with their region's needs.

\section{Acknowledgment}

This work and the authors' response plan's success also included the invaluable leadership of Adam Keene, Charles Esenwa, David Altschul, Elodia Mercer, Emad Eskandar, Mark Mehler, Rishi Malhotra, and Solomon Moshé. They specially thank all their neuroscience colleagues in the Montefiore Health System, clinical house staff, nursing, ancillary support staff, administrators, and, of course, their patients and their families. The authors have all been supported by great teachers and colleagues. They dedicate this work in the solemn memory of Dr. James T. Goodrich and the many others lost in this crisis.

\section{Study funding}

No targeted funding reported.

\section{Disclosure}

The authors report no disclosures relevant to the manuscript. Go to Neurology.org/N for full disclosures.

\section{Publication history}

Received by Neurology May 27, 2020. Accepted in final form July 21, 2020.

Appendix Authors

\begin{tabular}{lll}
\hline Name & Location & Contribution \\
\hline $\begin{array}{l}\text { Daniel J. } \\
\text { Correa, MD, }\end{array}$ & $\begin{array}{l}\text { Montefiore } \\
\text { MSc }\end{array}$ & $\begin{array}{l}\text { Corresponding author; designed } \\
\text { Bronx, NY }\end{array}$ \\
& $\begin{array}{l}\text { and conceptualized the response } \\
\text { plan and manuscript; major role in } \\
\text { the acquisition and analysis of data; } \\
\text { and drafted and edited the } \\
\text { manuscript for intellectual content }\end{array}$ \\
& \\
\hline
\end{tabular}

\begin{tabular}{|c|c|c|}
\hline $\begin{array}{l}\text { Daniel L. } \\
\text { Labovitz, } \\
\text { MD }\end{array}$ & $\begin{array}{l}\text { Montefiore } \\
\text { Medical Center, } \\
\text { Bronx, NY }\end{array}$ & $\begin{array}{l}\text { Designed and conceptualized the } \\
\text { response plan and manuscript; } \\
\text { analyzed the data; and drafted and } \\
\text { edited the manuscript for } \\
\text { intellectual content }\end{array}$ \\
\hline
\end{tabular}

\begin{tabular}{lll}
\hline Mark J. & Montefiore & $\begin{array}{l}\text { Designed and conceptualized the } \\
\text { Milstein, }\end{array}$ \\
MD & Medical Center, & $\begin{array}{l}\text { response plan and manuscript; } \\
\text { drafted portions of the manuscript; } \\
\text { and editing and revisions of the final } \\
\end{array}$ \\
& manuscript for intellectual content
\end{tabular}

\begin{tabular}{lll}
\hline $\begin{array}{l}\text { Renee } \\
\text { Monderer, } \\
\text { MD }\end{array}$ & $\begin{array}{l}\text { Montefiore } \\
\text { Medical Center, } \\
\text { Bronx, NY }\end{array}$ & $\begin{array}{l}\text { Designed and conceptualized the } \\
\text { response plan and manuscript and } \\
\text { editing and revisions of the final } \\
\text { manuscript for intellectual content }\end{array}$ \\
\hline $\begin{array}{l}\text { Sheryl R. } \\
\text { Haut, MD, } \\
\text { MSc }\end{array}$ & $\begin{array}{l}\text { Montefiore } \\
\text { Medical Center, }\end{array}$ & $\begin{array}{l}\text { Senior author; designed and } \\
\text { conceptualized the response plan } \\
\text { and manuscript; analyzed the data; } \\
\text { and drafted and edited the } \\
\text { manuscript for intellectual content }\end{array}$ \\
\hline
\end{tabular}

\section{References}

1. Health NDo. COVID-19: Data [online]. Available at: 1.nyc.gov/site/doh/covid/ covid-19-data-archive.page. Accessed April 21, 2020.

2. Leira EC, Russman AN, Biller J, et al. Preserving stroke care during the COVID-19 pandemic: potential issues and solutions. Neurology 2020;95:124-133.

3. Shellhaas RA. Neurologists and COVID-19: a note on courage in a time of uncertainty. Neurology 2020;94:855-857.

4. Lee IK, Wang CC, Lin MC, Kung CT, Lan KC, Lee CT. Effective strategies to prevent coronavirus disease-2019 (COVID-19) outbreak in hospital. J Hosp Infect 2020;105: 102-103.

5. Galanopoulou AS, Ferastraoaru V, Correa DJ, et al. EEG findings in acutely ill patients investigated for SARS-CoV-2/COVID-19: a small case series preliminary report. Epilepsia Open 2020;5:314-324.

6. Hollander JE, Carr BG. Virtually perfect? Telemedicine for Covid-19. N Engl J Med 2020;382:1679-1681.

7. President Trump Expands Telehealth Benefits for Medicare Beneficiaries During COVID-19 Outbreak. Centers for Medicare \& Medicaid Services, 2020.

8. General Provider Telehealth and Telemedicine Tool Kit [online]. 2020. Available at: cms.gov/files/document/general-telemedicine-toolkit.pdf. Accessed March 20, 2020.

9. Grossman SN, Han SC, Balcer LJ, et al. Rapid implementation of virtual neurology in response to the COVID-19 pandemic. Neurology 2020;94:1077-1087.

10. Antoniello D, Nisar T, Kirchoff-Torres KFC, Natalie T, Liberman AL, Labovitz DL. Invited Commentary: stroke assessment in the COVID-19 era: the observational neurologic exam. In: Callaghan BC, Kerber KA, eds. COVID-19 (Coronavirus) Innovations in Care Delivery: Neurology Blogs: American Academy of Neurology, 2020.

11. FAQs on Telehealth and HIPAA during the COVID-19 Nationwide Public Health Emergency [online]. 2020. Available at: hhs.gov/sites/default/files/telehealth-faqs508.pdf. Accessed March 19, 2020.

12. Chen $\mathrm{T}, \mathrm{Wu} \mathrm{D}$, Chen $\mathrm{H}$, et al. Clinical characteristics of 113 deceased patients with coronavirus disease 2019: retrospective study. BMJ 2020;368:m1091.

13. Mao L, Jin H, Wang M, et al. Neurologic manifestations of hospitalized patients with coronavirus disease 2019 in Wuhan, China. JAMA Neurol 2020;77:683-690.

14. Poyiadji N, Shahin G, Noujaim D, Stone M, Patel S, Griffith B. COVID-19-associated acute hemorrhagic necrotizing encephalopathy: CT and MRI features. Radiology 2020:201187.

15. Moriguchi T, Harii N, Goto J, et al. A first case of meningitis/encephalitis associated with SARS-Coronavirus-2. Int J Infect Dis 2020;94:55-58. 
16. Waldman G, Mayeux R, Claassen J, et al. Preparing a neurology department for SARSCoV-2 (COVID-19): early experiences at Columbia University Irving Medical Center and the New York Presbyterian Hospital in New York City. Neurology 2020;94: 886-891.

17. Yang L, Brown-Johnson CG, Miller-Kuhlmann R, et al. Accelerated launch of video visits in ambulatory neurology during COVID-19: key lessons from the Stanford experience. Neurology 2020;95:305-311.

18. French JA, Brodie MJ, Caraballo R, et al. Keeping people with epilepsy safe during the COVID-19 pandemic. Neurology 2020;94:1032-1037.
19. Rosenbaum L. Facing Covid-19 in Italy-ethics, logistics, and therapeutics on the epidemic's front line. N Engl J Med 2020;382:1873-1875.

20. Spina S, Marrazzo F, Migliari M, Stucchi R, Sforza A, Fumagalli R. The response of Milan's Emergency Medical System to the COVID-19 outbreak in Italy. Lancet 2020; 395:e49-e50.

21. Fagiuoli S, Lorini FL, Remuzzi G. Covid-19 Bergamo Hospital Crisis Unit. Adaptations and lessons in the province of Bergamo. N Engl J Med 2020;382:e71.

22. Remuzzi A, Remuzzi G. COVID-19 and Italy: what next?. Lancet 2020;395: $1225-1228$.

\section{NEW EPISODE}

\section{ค}

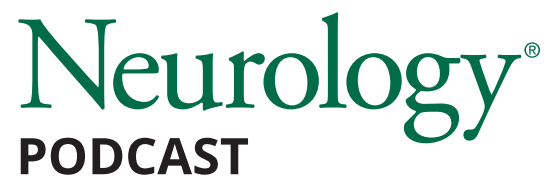

September 29, 2020

\section{Ocular motor measures of visual processing changes in visual snow syndrome (see p. 569)}

In the first segment, Dr. Teshamae Monteith and Dr. Joanne Fielding talk about Dr. Fielding's paper discussing visual processing in visual snow syndrome. Then, Dr. Jeffrey Ratliff speaks with Dr. Sonia Vallabh about her life and work in prion disease. Dr. Vallabh has been profiled in WIRED and the New York Times.

Disclosures can be found at Neurology.org.

CME Opportunity: Listen to this week's Neurology Podcast and earn 0.5 AMA PRA Category 1 CME Credits ${ }^{\mathrm{TM}}$ by completing the online Podcast quiz.

\section{Visit the Neurology ${ }^{\circledR}$ Resident \& Fellow Website}

Click on Residents \& Fellows tab at Neurology.org.

Now offering:

- Neurology ${ }^{\circledR}$ Resident \& Fellow Editorial team information

- "Search by subcategory" option

- E-pearl of the Week

- RSS Feeds

- Direct links to Continuum ${ }^{\circledR}$, Career Planning, and AAN Resident \& Fellow pages

- Recently published Resident \& Fellow articles

- Podcast descriptions

- Blogs by Editors and Resident \& Fellow team members

f Find Neurology ${ }^{\circledR}$ Residents \& Fellows Section on Facebook: facebook.com/AANResidentsAndFellows

Follow Neurology ${ }^{\circledR}$ on Twitter: @GreenJournal \#NeurologyRF

(ㄱ) Find Neurology ${ }^{\circledR}$ Residents \& Fellows Section on Instagram: @aanbrain \#NeurologyRF 


\section{Neurology}

Folding a neuroscience center into streamlined COVID-19 response teams: Lessons in

Daniel J. Correa, Daniel L. Labovitz, Mark J. Milstein, et al.

Neurology 2020;95;583-592 Published Online before print July 30, 2020

DOI 10.1212/WNL.0000000000010542

This information is current as of July 30, 2020

Updated Information \&

Services

References

Subspecialty Collections

Permissions \& Licensing

Reprints including high resolution figures, can be found at:

http://n.neurology.org/content/95/13/583.full

This article cites 16 articles, 7 of which you can access for free at: http://n.neurology.org/content/95/13/583.full\#ref-list-1

This article, along with others on similar topics, appears in the following collection(s):

All Health Services Research

http://n.neurology.org/cgi/collection/all_health_services_research Health systems

http://n.neurology.org/cgi/collection/health_systems

Patient safety

http://n.neurology.org/cgi/collection/patient_safety

Information about reproducing this article in parts (figures,tables) or in its entirety can be found online at:

http://www.neurology.org/about/about_the_journal\#permissions

Information about ordering reprints can be found online:

http://n.neurology.org/subscribers/advertise

Neurology ${ }^{\circledR}$ is the official journal of the American Academy of Neurology. Published continuously since 1951, it is now a weekly with 48 issues per year. Copyright @ 2020 American Academy of Neurology. All rights reserved. Print ISSN: 0028-3878. Online ISSN: 1526-632X.

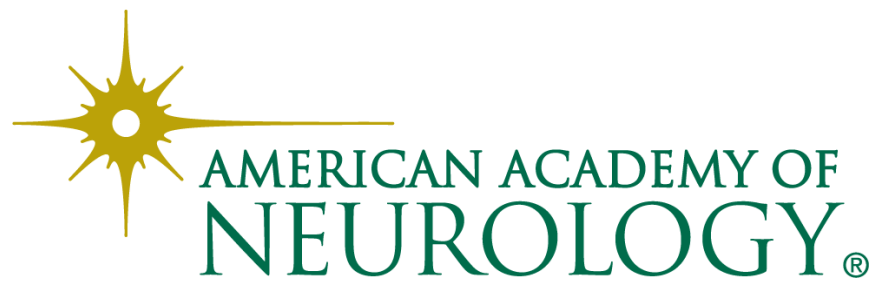

